\title{
SYMMETRY BREAKING AND BIFURCATIONS IN COMPLEX LORENZ MODEL
}

\author{
A. D. Kiselev \\ Department of Pure and Applied Mathematics, Chernihiv Technological Institute, \\ 95 Shevchenko Str., UA-250027, Chernihiv, Ukraine \\ E-mail:kisel@elit.chernigov.ua; adk@kid.ti.chernigov.ua
}

(Received July 14, 1997)

\begin{abstract}
The concept of broken symmetry is used to study stability of equilibrium and time doublyperiodic bifurcating solutions of the complex nonresonant Lorenz model as a function of the frequency detuning on the basis of modified Hopf theory. By contrast to the well-known real Lorenz equations, the system in question is invariant under the action of Lie group transformations (rotations in complex planes) and an invariant set of stationary points is found to bifurcate into an invariant torus, which is stable under the detuning exceeding its critical value. If the detuning then goes downward numerical analysis reveals that after a cascade of period-doublings the strange Lorenz attractor is formed in the vicinity of zero detuning.
\end{abstract}

Key words: broken symmetry, bifurcations, chaos, Lorenz model.

PACS number(s): 05.45.+b, 42.60.Mi, 42.65.Sf, 82.20.Mj

\section{INTRODUCTION}

The well-known Lorenz equations: $\dot{X}=\sigma(-X+$ $Y), \dot{Y}=r X-Y+X Z, \dot{Z}=-b Z-X Y$ were originally derived in [1] and have been studied intensively for the last two decades (see, for example, [2-6] and references therein). The above system (which will be referred as the real Lorenz model for $X(t), Y(t)$ and $Z(t)$ that are real-valued functions) was obtained from a set of hydrodynamic equations in the three-mode approximation to describe the convective motion of a layer of fluid that is warmer at the bottom than at the top, so that $\sigma$ is the Prandtl number; $r$ (controlling parameter) is the Rayleigh number and is proportional to the temperature difference; $b$ depends on geometrical properties of the fluid layer.

One of the most striking features of the real Lorenz model is the appearance of so-called strange Lorenz attractor under the controlling parameter $r$ exceeding its critical value $r_{c}=\frac{\sigma+b+3}{\sigma-b-1} \sigma$ ( $\sigma$ must be larger than $b+1)$. The term 'strange attractor' (or 'chaotic attractor') is commonly used for an attracting set that has a rather complicated structure and each trajectory within the attractor is exponentially unstable. There is a number of different quantities to measure the complexity (stochasticity) of the attractor structure: capacity (fractal dimension), information dimension, Hausdorff and Liapunov (Kaplan-Yorke formula) dimensions, K-entropy and so on.

Apparently, an understanding of the reasons for the occurence of the chaotic behavior in dissipative nonlinear dynamical systems such as the real Lorenz model requires the study of bifurcations that produces qualitative changes in the phase portrait of a system.

Since in this paper we are primary concerned with the complex Lorenz model that can be regarded as a gener- alization of the real Lorenz system (see below for details) and our purpose is to study stability of certain bifurcating solutions of the system on the basis of bifurcation analysis, it is of interest to discuss some relevant results for the real Lorenz system.

Let us begin with stability and bifurcations of equilibria (steady states). The null steady state is given by $X=Y=Z=0$ and is asymptotically stable at $r<1$. This solution loses its stability at $r=1$ and there are two asymptotically stable bifurcating steady states $X=Y= \pm \sqrt{b(r-1)}, Z=r-1$ provided that $1<r<r_{c}$. So we have two stationary points bifurcated from the zero state at $r=1$ that correspond to the initiation of a convective flow. Note that the linearized operator governing stability of the solutions in question (Liapunov's first theorem) has a pair of complex conjugate eigenvalues with negative real parts in the neighborhood of $r_{c}$. These eigenvalues become pure imaginary at $r=r_{c}$, so that the steady states are unstable under $r>r_{c}$. It follows that the Hopf bifurcation theory [4] can be applied to show the existence of the bifurcating time periodic solution. Stability of this branching solution is determined by the Floquet exponents: the solution is stable (unstable) if it appears supercritically (subcritically). In the case under consideration the bifurcation is found to be subcritical [7]. This result lends support to the view of 'drastic' conditions for the occurence of chaos in the real Lorenz model.

Detailed description of how a strange Lorenz attractor forms is beyond the scope of this paper. In brief, this can be understood as being due to the occurrence of a homoclinic orbit in the system: as $r$ passes through the value at which the homoclinic 'explosion' takes place, a strange invariant set of trajectories is produced, including an infinite number of periodic orbit $[2,5,6]$. Note that, in addition, the real Lorenz model is known to exhibit period-doubling [8], intermittency [9] and hysteresis 
[10] in various ranges of its parameter space.

There are several physical problems leading to complexification of the Lorenz equations [11] where some of the functions are complex-valued. As an example, we shall keep in mind, the simplest one-mode laser equations obtained in the semiclassical approximation [12]:

$$
\left\{\begin{array}{l}
\dot{b}=-(\kappa+i \omega) \cdot b-i g \cdot \alpha \\
\dot{\alpha}=-\left(\gamma+i \omega_{a}\right) \cdot \alpha+i g \cdot b \cdot S \\
\dot{S}=\left(d_{0}-S\right) / T-4 g \cdot \Im(\alpha \cdot \bar{b})
\end{array}\right.
$$

where $b$ is the dimensionless complex amplitude of the electromagnetic field mode; $\omega(\kappa)$ is the frequency (the relaxation constant) of the mode; $\alpha$ is the dimensionless dipole transition matrix element; $S$ is the inversion of the atomic level population; $\omega_{a}$ is the frequency of the atomic transition; $d_{0}$ is the parameter characterizing the intensity of pumping; $g$ is the coupling constant; $\gamma\left(T^{-1}\right)$ is the transverse (longitudinal) relaxation constant.

After making the substitutions:

$$
\begin{gathered}
t \rightarrow \gamma t, b=\gamma z_{1} /(2 g) \\
\alpha=i S_{0} z_{2} / 2, S=d_{0}+S_{0} z_{3}, S_{0}=\gamma \kappa / g^{2}
\end{gathered}
$$

and going over to the interaction representation the system (1) can be rewritten as the complex Lorenz model [11]:

$$
\left\{\begin{array}{l}
\dot{z_{1}}=\sigma\left(-(1+i \Delta) \cdot z_{1}+z_{2}\right) \\
\dot{z_{2}}=-(1-i \Delta+i \rho) \cdot z_{2}+(r+i \rho) \cdot z_{1}+z_{1} z_{3} \\
\dot{z_{3}}=-b z_{3}-\Re\left(z_{1} \cdot \bar{z}_{2}\right)
\end{array}\right.
$$

where $\sigma=\kappa / \gamma, r=d_{0} / S_{0}, b=(\gamma T)^{-1}, \rho=0, \Delta=$ $\left(\omega_{a}-\omega\right) /(\kappa+\gamma)$ is the frequency detuning. Here the parameter $\rho$ is introduced to make the system identical with those considered in [11].

Note that $z_{1}(t)$ and $z_{2}(t)$ are complex-valued functions, so that the system (2) consists of five real equations.

In this paper we are aimed to study how the detuning $\Delta$ influences bifurcations of equilibrium and doublyperiodic solutions in the complex Lorenz model (eq. (2)).

Since, by contrast to the real Lorenz model, the system (2) has a continuous symmetry group (Lie group of rotations in complex planes) we approach the problem of detuning induced effects within the unified concept of symmetry breaking.

In Sec. 2 it is shown that due to the symmetry the null equilibrium state of the complex Lorenz model (spontaneous emission) bifurcates into an invariant set of stationary points. Stability of the equilibrium states is studied as a function of detuning. It is found that under $\sigma>b+1$ there is a critical value of the controlling parameter $r$ (pumping intensity), $r_{c}$, such that the states of the invariant set become exponentially unstable at $r>r_{c}$ and $r_{c}$ is an increasing function of $\Delta^{2}$.
In Sec. 3 analytical power series Hopf technique is extended on the system invariant under the action of a continuous symmetry group to construct bifurcating solutions and to investigate their stability in the vicinity of $r=r_{c}$ at $\Delta \neq 0$. Due to the symmetry breaking the bifurating solution is appeared to be doubly-periodic at $\Delta \neq 0$. It means that nonzero detuning results in the appearance of low-frequency Goldstone-type mode related to the motion along an orbit of the group. Moreover, the broken symmetry is found to affect stability of the branching solution.

The results of numerical analysis and concluding remarks are given in Sec. 4. The dependence of the relevant Floquet exponent on $\Delta$ is calculated. It is obtained that there is a critical detuning $\Delta_{c}$ such that the bifurcating doubly-periodic solution is stable at $|\Delta|>\Delta_{c}$. As a consequence, there are different routes to chaos depending on the detuning.

\section{SYMMETRY AND BIFURCATIONS OF EQUILIBRIA}

Taking $r$ as a bifurcation (controlling) parameter, eq. (2) can be rewritten as an autonoumous dynamical system with quadratic nonlinearity:

$$
\dot{\mathbf{x}}=\mathbf{f}(\mathbf{x})=L \mathbf{x}+\mathbf{f}_{2}(\mathbf{x}, \mathbf{x})
$$

where

$$
\begin{gathered}
\mathbf{x}=\left(x_{1}, x_{2}, x_{3}, x_{4}, x_{5}\right) \\
z_{1}=x_{1}+i x_{2}, z_{2}=x_{3}+i x_{4}, z_{3}=x_{5}, \\
L=D \mathbf{f}(0)\left(L_{i j}=\frac{\partial f_{i}}{\partial x_{j}}(0)\right) \\
f_{2}^{i}(\mathbf{x}, \mathbf{y})=\sum_{n, m} \frac{\partial^{2} f_{i}}{\partial x_{n} \partial x_{m}} \cdot x_{n} \cdot y_{m}
\end{gathered}
$$

Clearly, system (2) is invariant under the transformations:

$$
z_{1} \rightarrow \exp (i \phi) \cdot z_{1}, z_{2} \rightarrow \exp (i \phi) \cdot z_{2}, z_{3} \rightarrow z_{3}
$$

so that eq. (3) has a continuous symmetry group $G$ of rotations in the $x_{1}-x_{2}$ and $x_{3}-x_{4}$ planes, $G \sim U(1)$ :

$$
\mathbf{f}(T(\phi) \cdot \mathbf{x})=T(\phi) \cdot \mathbf{f}(\mathbf{x}), \quad T(\phi) \in G .
$$

Note that there is an additional discrete symmetry in the complex Lorenz model:

$$
z_{1} \rightarrow \bar{z}_{1}, z_{2} \rightarrow \bar{z}_{2}, z_{3} \rightarrow z_{3}, \Delta \rightarrow-\Delta, \rho \rightarrow-\rho
$$




\section{A. D. KISELEV}

The latter implies no dependence on the sign of detuning at $\rho=0$. (In what follows we shall concentrate on this case, assuming, for brevity, that $\Delta \geq 0$.)

Eq. (4) gives

$$
\mathbf{f}\left(T(\phi) \cdot \mathbf{x}_{s t}\right)=0 \Rightarrow\left|D \mathbf{f}\left(\mathbf{x}_{s t}\right)\right|=0
$$

where $\mathbf{x}_{s t}$ is a noninvariant equilibrium solution to eq. (3) and its orbit $T(\phi) \cdot \mathbf{x}_{s t}$ produces an invariant set of equilibria. The second equation in (5) is obtained by differentiating the first one with respect to $\phi$. Along this line we come to the conclusion that the matrix of the first approximation, that govern linearized stability of $\mathbf{x}_{s t}$, is degenerate and its eigenvector (null vector) for the zero eigenvalue is $\mathbf{e}_{0}=K \cdot \mathbf{x}_{s t}$, where $K=\left.\frac{d}{d \phi} T(\phi)\right|_{\phi=0}$ is the generator of $G$.

It can be readily shown that the null stationary point related to the regime of spontaneous emission loses its stability after the pumping intensity exceeded its critical value

$$
r>1+\Delta^{2}
$$

and the solution bifurcated from the zero state can be taken in the form

$$
\begin{aligned}
& x_{1}^{s t}=x_{3}^{s t}=q=\sqrt{b \cdot\left(r-1-\Delta^{2}\right)}, \\
& x_{2}^{s t}=0, x_{4}^{s t}=\Delta \cdot q, x_{5}=1+\Delta^{2}-r .
\end{aligned}
$$

According to eq. (5), the stationary point (7) provides an invariant set of the steady states corresponding to the appearance of coherent light emission (laser generation). In the first approximation stability of the state (7) is determined by eigenvalues of the following matrix:

$$
D \mathbf{f}\left(\mathbf{x}_{s t}\right)=L_{c}=\left(\begin{array}{ccccc}
-\sigma & \Delta \cdot \sigma & \sigma & 0 & 0 \\
-\Delta \cdot \sigma & -\sigma & 0 & \sigma & 0 \\
1+\Delta^{2} & 0 & -1 & -\Delta & q \\
0 & 1+\Delta^{2} & \Delta & -1 & 0 \\
q & -\Delta \cdot q & -q & 0 & -b
\end{array}\right)
$$

As it can be seen from eq. (5), $\left|L_{c}\right|=0$ and the kernel of $L_{c}$ is defined by the null vector $\mathbf{e}_{0}=(0,1,-\Delta, 1,0)$. If all other four eigenvalues have negative real parts, then symmetry arguments combined with Liapunov theorem lead to the conclusion that the set of stationary points is asymptotically stable. Obviously, the steady state on the orbit cannot enjoy the property of being asymptotically stable for there is another steady state in any of its neighborhoods. So, in this case we have the asymptotically stable invariant set of stable stationary points. Some straightforward algebra on the subject was made in [13] for $\Delta=0$.

If $\sigma>b+1$ (this condition is found to be independent of $\Delta$ and $\rho$ ), there is a critical value of the bifurcation parameter, such that the solution in question is unstable at $r>r_{c}$ and $L_{c}$ has a pair of complex conjugate imaginary eigenvalues $\lambda_{1}=\bar{\lambda}_{2}=i \alpha$ at $r=r_{c}$. Equations for $r_{c}$ and $\alpha$ can be derived by making use of Routh-Hurwitz criteria:

$$
\begin{aligned}
p_{1} p_{2} p_{3} & =p_{1}^{2}+p_{0} p_{3}^{2}, \\
\alpha^{2} & =\frac{p_{1}}{p_{3}},
\end{aligned}
$$

where $p_{i}$ are coefficients of the characteristic polynomial:

$$
\begin{aligned}
& p_{0}=2 \sigma b(1+\sigma)\left(r-1-\Delta^{2}\right), \\
& p_{1}=b\left(\left(1+\Delta^{2}\right) \sigma^{2}+3 \sigma(r-2)+r\right), \\
& p_{2}=(\sigma+1)^{2}+1+r+2 \sigma+\Delta^{2}\left((\sigma-1)^{2}-b\right), \\
& p_{3}=2 \sigma+b+2 .
\end{aligned}
$$

In addition, it can be obtained that $\Re\left(\left.\frac{d}{d r} \lambda_{1}\right|_{r=r_{c}}\right)>0$ and then, if not symmetry induced degeneracy of $L_{c}$, we could meet all conditions for the occurence of the Hopf bifurcation.

\section{HOPF-TYPE BIFURCATION}

There are different techniques to investigate Hopf bifurcations and stability of bifurcating time periodic solutions [4]. In this section we adopt the perturbative method, closely related to the analytical technique by Hopf, to explicitly construct the bifurcating solution in the form of power series over small parameter $\epsilon$ characterizing amplitude of the solution in the neighborhood of the bifurcation point $r=r_{c}[4,14]$. Coefficients of the power series can be derived by making use of Fredholm alternative and linearized stability of the solution, determined by the Floquet exponents which can be studied on the basis of the factorization theorem [15].

In trying to make analysis along the above line we need to modify the method to bypass mathematical difficulties arising from the degeneracy of $L_{c}$. To avoid equations that have no solutions it is assumed that the symmetry is 'spontaneously' broken and the bifurcating solution is taken in the form:

$$
\mathbf{x}=T(\epsilon \theta) \cdot \mathbf{u}(t)=T(\epsilon \theta) \cdot\left(\mathbf{x}_{s t}+\epsilon \cdot \mathbf{z}(t)\right)
$$

Substitution of eq. (15) in eq. (3) gives the equation for $\mathbf{u}(t)$ :

$$
\dot{\mathbf{u}}+\epsilon \cdot K \mathbf{u}=\mathbf{f}(\mathbf{u})
$$

Let us introduce the renormalized frequency $\Omega(\epsilon)=$ $\alpha /(1+\tau(\epsilon))$, so that $\mathbf{z}(s)=\mathbf{z}(\Omega t)$ is a $2 \pi$-periodic vectorvalued function. The equation for $\mathbf{z}(s)$ reads 


$$
\begin{aligned}
& \alpha \cdot\left(\dot{\mathbf{z}}+\dot{\theta} \cdot\left(\mathbf{e}_{0}+\epsilon \cdot K \cdot \mathbf{z}\right)\right) \\
& =(1+\tau) \cdot\left(L_{c} \mathbf{z}+\epsilon \mathbf{f}_{2}(\mathbf{z}, \mathbf{z})\right)
\end{aligned}
$$

where $L_{c}$ is defined by eq. (8) and the dot stands for the derivative with respect to $s$. The $2 \pi$-periodic solution of eq. (17) can be found in the form of power series in $\epsilon$ :

$$
\begin{aligned}
& \mathbf{z}(s)=\sum_{n=0}^{\infty} \mathbf{z}_{n}(s) \epsilon^{n}, \quad \tau=\sum_{n=1}^{\infty} \tau_{n} \epsilon^{n}, \\
& \dot{\theta}=\sum_{n=0}^{\infty} \omega_{n} \epsilon^{n} .
\end{aligned}
$$

As is seen from eqs. $(7,8)$, the quantity $q=$ $\sqrt{b \cdot\left(r-1-\Delta^{2}\right)}$ can be conveniently chosen as a bifurcation parameter, so that

$$
\begin{aligned}
q & =q_{c}+\Delta q=q_{c}+\sum_{n=1}^{\infty} q_{n} \epsilon^{n}, \\
L_{c} & =L_{c}^{(0)}+L_{c}^{(1)} \cdot \Delta q, \\
\mathbf{e}_{0} & =K \mathbf{x}_{s t}=\mathbf{e}_{0}^{(0)}+\mathbf{e}_{0}^{(1)} \cdot \Delta q,
\end{aligned}
$$

where $L_{c}^{(0)}, \mathbf{e}_{0}^{(0)}$ are $L_{c}, \mathbf{e}_{0}$ at $q=q_{c}$. Recall that $L_{c}^{(0)}$ has a pair of pure imaginary complex conjugate eigenvalues: $L_{c}^{(0)} \mathbf{e}_{1}=i \alpha \mathbf{e}_{1}, L_{c}^{(0)} \mathbf{e}_{2}=-i \alpha \mathbf{e}_{2}$. (For brevity, the superscript (0) will be omitted from the eigenvector notations.) Note that eigenvectors $\left\{\mathbf{e}_{0}^{*}, \ldots \mathbf{e}_{5}^{*}\right\}$ of the operator adjoint to $L_{c}^{(0)}$ and $\left\{\mathbf{e}_{0}, \ldots \mathbf{e}_{5}\right\}$ are mutually orthogonal: $\left\langle\mathbf{e}_{i}^{*}, \mathbf{e}_{j}\right\rangle=\delta_{i j}$.

According to the standard perturbative technique, the insertion of eqs. (18-21) into eq. (17) yields the equations to be solved for $\mathbf{z}_{n}(s)$ successively. In the case of $n>0$, the equations can be written in the form:

$$
\begin{aligned}
& \left\{\alpha \cdot \frac{d}{d s}-L_{c}^{(0)}\right\} \mathbf{z}_{n}(s) \\
& =\mathbf{f}^{(n)}(s)=\mathbf{f}_{0}^{(n)}+\sum_{k=1}^{2 n-2}\left[\mathbf{f}_{k}^{(n)} \cdot \exp i k s+c . c .\right] .
\end{aligned}
$$

Parameters $q_{n}, \omega_{n}$ and $\tau_{n}$ can be found by making use of Fredholm alternative. The latter states that eq. (22) has $2 \pi$-periodic solutions if and only if

$$
\begin{aligned}
& \left\langle\mathbf{e}_{0}^{*}, \mathbf{f}_{0}^{(n)}\right\rangle=0 \\
& \left\langle\mathbf{e}_{1}^{*}, \mathbf{f}_{1}^{(n)}\right\rangle=0 .
\end{aligned}
$$

Note that eq. (23) is the solvability condition for the time independent part of $\mathbf{z}_{n}(s)$ and eq. (24) is to get rid of the so-called secular terms.

In order to reduce the remaining arbitrariness in choice of the solutions, $\mathbf{z}_{n}(s)$ are subjected to the following additional constraints:

$$
\left\langle\mathbf{e}_{0}^{*}, \mathbf{z}_{n}(s)\right\rangle=0
$$

$$
\int_{0}^{2 \pi}\left\langle\mathbf{e}_{1}^{*}, \mathbf{z}_{n}(s)\right\rangle \exp (-i s) d s=0, n>0
$$

In the zero-order approximation we have

$$
\left\{\alpha \cdot \frac{d}{d s}-L_{c}^{(0)}\right\} \mathbf{z}_{0}(s)=-\alpha \omega_{0} \mathbf{e}_{0}
$$

so that

$$
\mathbf{z}_{0}(s)=A \cdot \mathbf{e}_{1} \exp (i s)+c . c ., \omega_{0}=0
$$

where $A$ is a complex integration constant that is determined by the initial condition for $\mathbf{z}_{0}(s)$ and can be eliminated from the consideration by renormalizing the eigenvector $\mathbf{e}_{1}$.

After some rather straightforward calculations the following results can be obtained:

$$
\begin{gathered}
q_{2 n+1}=\tau_{2 n+1}=\omega_{2 n}=0, \\
\alpha \cdot \omega_{1}=2 \cdot|A|^{2} \cdot\left\langle\mathbf{e}_{0}^{*}, \mathbf{f}_{2}\left(\mathbf{e}_{1}, \mathbf{e}_{2}\right)\right\rangle, \\
\mathbf{z}_{1}(s)=\mathbf{b}_{0}+\left\{A^{2} \cdot \mathbf{b}_{1} \cdot \exp (2 i s)+c . c .\right\} \\
-q_{2} \cdot \Re\left(\frac{\partial}{\partial q} \lambda_{c}\right)=\Re(k)
\end{gathered}
$$

where $\lambda_{c}$ is the eigenvalue of $L_{c}$, such that $\lambda_{c}=i \alpha$ at $q=q_{c}$,

$$
\begin{aligned}
& k=2 \cdot|A|^{2} \cdot\left\{2 \cdot\left\langle\mathbf{e}_{1}^{*}, \mathbf{f}_{2}\left(\mathbf{e}_{1}, \mathbf{b}_{0}\right)\right\rangle\right. \\
& \left.+\left\langle\mathbf{e}_{1}^{*}, \mathbf{f}_{2}\left(\mathbf{e}_{2}, \mathbf{b}_{1}\right)\right\rangle\right\}-\alpha \cdot \omega_{1} \cdot\left\langle\mathbf{e}_{1}^{*}, K \mathbf{e}_{1}\right\rangle
\end{aligned}
$$

and the vectors $\mathbf{b}_{0}, \mathbf{b}_{1}$ are solutions of the equations:

$$
\begin{gathered}
\left\{2 i \alpha-L_{c}^{(0)}\right\} \mathbf{b}_{1}=\mathbf{f}_{2}\left(\mathbf{e}_{1}, \mathbf{e}_{1}\right), \\
-L_{c}^{(0)} \mathbf{b}_{0}=\mathbf{f}_{2}\left(\mathbf{e}_{1}, \mathbf{e}_{2}\right)-\left\langle\mathbf{e}_{0}^{*}, \mathbf{f}_{2}\left(\mathbf{e}_{1}, \mathbf{e}_{2}\right)\right\rangle \cdot \mathbf{e}_{0} .
\end{gathered}
$$


At this stage we get the modification of Hopf's theory with the allowance for the symmetry breaking, so that bifurcating solution appears to be doubly periodic. In other words, there are two types of frequencies: the basic frequency $\Omega$ and the Goldstone-type low frequency mode with $\epsilon \dot{\theta} \sim \Delta^{2}$. (The latter can be inferred from eq. (30).) It is of interest to note that another distinctive feature of the above results is the last term of eq. (33). This term, being geometrical in nature, accounts for the broken symmetry and will be shown to be of importance for stability analysis.

In the Hopf theory the conclusion on stability of the bifurcating solution can be drawn from eqs. $(32,33)$ based on the factorization theorem $[4,14-15]$ that states about the stability depending on the sign of $\Re(k)$ : if $\Re(k)<0$, then bifurcation is supercritical and the time periodic branching solution is stable at $q>q_{c}$; if $\Re(k)>0$ the solution appears subcritical.

This raises the question as to whether the above result can be applied in the case under consideration. In the remaining part of the section we outline a way to recover the factorization theorem. The basic idea is to use an extended version of ansatz $[14,15]$ for the perturbation of $\mathbf{u}(s)$ :

$$
\mathbf{u}(s) \rightarrow \mathbf{u}(s)+\exp (\gamma s) \cdot \mathbf{v}(s)
$$

where $\gamma$ is the Floquet exponent and $\mathbf{v}(s)$ is the $2 \pi$ periodic part of the perturbation.

The linearization of eq. (16) gives an equation for $\mathbf{v}(s)$ :

$$
\gamma \cdot \mathbf{v}=\tilde{L} \cdot \mathbf{v}
$$

where $\tilde{L}=D \mathbf{f}(\mathbf{u})-\epsilon \cdot \dot{\theta} \cdot K-\Omega \cdot \frac{d}{d s}$.

In addition, we have

$$
\tilde{L} \cdot \dot{\mathbf{u}}=\tilde{L} \cdot K \mathbf{u}=0,
$$

$$
\begin{aligned}
& \tilde{L} \cdot \mathbf{u}^{\prime}=\Omega^{\prime} \cdot \dot{\mathbf{u}}+(\epsilon \dot{\theta})^{\prime} K \mathbf{u} \\
& +\epsilon \cdot q^{\prime} \cdot\left(\dot{\theta} \cdot K \cdot \mathbf{e}_{0}^{(1)}-L_{c}^{(1)} \cdot \mathbf{z}\right),
\end{aligned}
$$

where the prime stands for the partial derivative with respect to $\epsilon$.

Eqs. (38) is a direct consequence of the system invariance with respect to translations $s \rightarrow s+$ const (the system is autonomous) and under the action of the Lie group of symmetry.

In order to get $\epsilon$-expansion for the Floquet exponent $\gamma$ let us assume the following form of $\mathbf{v}(s)$ (the abovementioned ansatz):

$$
\mathbf{v}=\mathbf{u}^{\prime}+\beta_{1} \cdot \dot{\mathbf{u}}+\beta_{2} \cdot K \mathbf{u}+\epsilon \cdot q^{\prime} \cdot \tilde{\mathbf{v}}
$$

From this point onwards we can proceed in exactly the same way as in $[14,15]$ : equation for $\tilde{\mathbf{v}}$ is derived by substituting eq. (40) into eq. (37) and the use of eqs. (37, 39). The coefficients of the equation are given in terms of $\delta_{1}, \delta_{2}$ and $\delta_{3}: q^{\prime} \cdot \delta_{1}=\gamma \cdot \beta_{1}-\Omega^{\prime}, \epsilon \cdot q^{\prime} \cdot \delta_{2}=\gamma \cdot \beta_{2}-(\epsilon \dot{\theta})^{\prime}$, $\epsilon \cdot q^{\prime} \cdot \delta_{3}=\gamma$. After expanding of the coefficients and $\tilde{\mathbf{v}}$ in the power series over $\epsilon$, it is not difficult to derive the final result for $\gamma$ in the lowest order of $\epsilon$ :

$$
\Re(\gamma) \approx 2 \cdot \epsilon^{2} \cdot \Re(k)
$$

Thus, the factorization theorem is recovered by making use of the ansatz (40). It follows that eqs. $(32,33)$ are key equations for making conclusion on stability of the bifurcating invariant torus. In particular, eq. (41) implies that the torus is stable at $r>r_{c}$ under $\Re(k)<0$.

\section{NUMERICAL RESULTS AND DISCUSSION}

In the previous section we have studied how symmetry of the system affects Hopf-type bifurcation at $r=r_{c}$. Our findings are:

1. An invariant set of equilibria bifurcates into an invariant torus. In other words, the branching solution is time doubly-periodic, so that Goldstonetype low frequency mode is found to appear due to the symmetry breaking.

2. It is found that the factorization theorem holds in the case under consideration and the sign of $\Re(k)$ with $k$ defined by eq. (33) determines stability of the torus. The last symmetry induced term in eq. (33) implies that the broken symmetry affects stability of the branching solution.

Note that the frequency of the Goldstone-type mode as well as the last term of eq. (33) tend to zero as $\Delta \rightarrow 0$.

In fig. 1 the dependencies of $\Re(k)$ on detuning $(\Delta)$ for $\sigma=5$ (solid line) and $\sigma=10$ (dashed line) at $b=1$ are shown. It is seen that in both cases there is a critical detuning $\Delta_{c}$ at which $\Re(k)$ changes its sign, so that bifurcation being subcritical at $\Delta<\Delta_{c}$ becomes supercritical at $\Delta>\Delta_{c}$. The latter means that an invariant set of equilibria eq. (7), corresponding to the laser generation, bifurcates into the stable torus as $r$ passes through $r_{c}$ under $\Delta>\Delta_{c}$.

To get some idea of qualitative changes of the attractor structure in relation to the detuning, there are three trajectories in $3 \mathrm{D} \Re\left(z_{1}\right)-\Re\left(z_{2}\right)-z_{3}$ space in fig. 2 presented at $\sigma=5, b=1$ and $r=r_{c}+0.2$ for various values of $\Delta$. fig. 2 a clearly reveales the attractor as an invariant torus at $\Delta=0.5>\Delta_{c} \approx 0.41$, whereas we have a well-known Lorenz attractor under $\Delta=0$ (fig. 2c). As is shown in fig. $2 \mathrm{~b}$, the intermediate case of $\Delta=0.1$ corresponds to an entangled structure which is hard to interpret. 


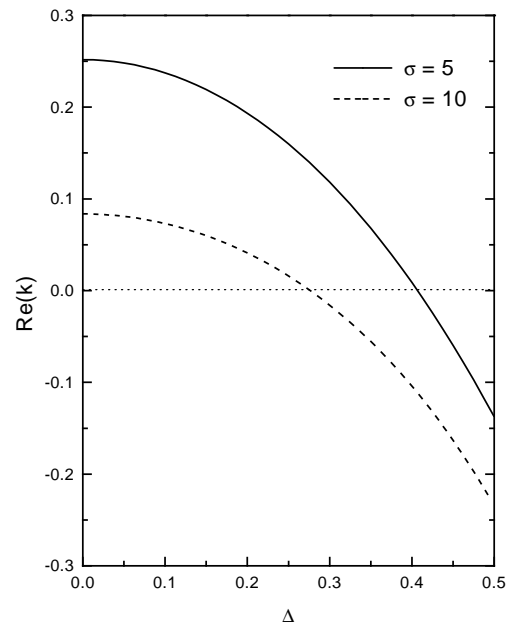

Fig. 1. Dependence of $\Re(k)$ (see eq. (33)) on $\Delta$ at $b=1$ for $\sigma=5$ (solid line) and $\sigma=10$ (dashed line). In both of the cases the coefficient is shown to change its sign provided the detuning exceeded its critical value.

One of the ways to clarify the point is to look at the relevant Fourier spectra. To this end, the Fourier spectra $\left|z_{1}(\omega)\right|$ and $\left|z_{3}(\omega)\right|$ are calculated at $\Delta=0.5$ (fig. 3 ) and $\Delta=0.1$ (fig. 4). Notice that $\left|z_{1}(\omega)\right|^{2}$ is proportional to the power spectrum of the electromagnetic field.

The Fourier spectrum $\left|z_{1}(\omega)\right|$ for $\Delta=0.5$, depicted in fig. 3 , indicates the high frequency peak at $\omega \approx \alpha=5.5$ and the two intensive low frequency peaks at $\omega=0$ and $\omega \approx 0.04$. Since the frequency $\omega \approx 0.04$ does not contribute to the spectrum $\left|z_{3}(\omega)\right|$, this peak can be attributed to the Goldstone-type mode. So, the numerical results are in agreement with the ones obtained from the theoretical analysis of Sec. 3. As far as the numerical analysis is concerned, it should be emphasized that, working with a relatively small number of points (less than 20000), we are not to present the results of high precision calculations, but our calculations has been made with reasonable accuracy for the investigation of the theoretical predictions qualitatively.

Coming back to fig. $2 \mathrm{~b}$ and looking at the Fourier spectra given in fig. 4, let us recall that, according to the theory of Sec. 3 and fig. 1, the relevant Floquet exponent is pure imaginary at $\Delta=\Delta_{c}$. So changing $\Delta$ from the above $\Delta_{c}$, where the invariant $2 \mathrm{D}$ torus is stable, downward ( $r$ is fixed) we encounter another bifurcation point at $\Delta=\Delta_{c}$, and the torus is expected to bifurcate into a $3 \mathrm{D}$ torus, embedded in the $5 \mathrm{D}$ phase space of the complex Lorenz model. Computer simulation confirms this conclusion. Comprehensive analysis of this secondary bifurcation is beyond the scope of this paper and will be published elsewhere. (Some results on the subject were obtained in [16]). A further decrease of $\Delta$ would result in other bifurcations. Taking into account that the spectra of fig. 4 are typical of period doublings, it can be suggested that the chaotic attractor forms at relatively small $\Delta$ after a cascade of doublings. The irregular Fourier spectrum at $\Delta=0.05$, displayed in fig. 5 , is clearly associated with chaotic dynamics of the system.

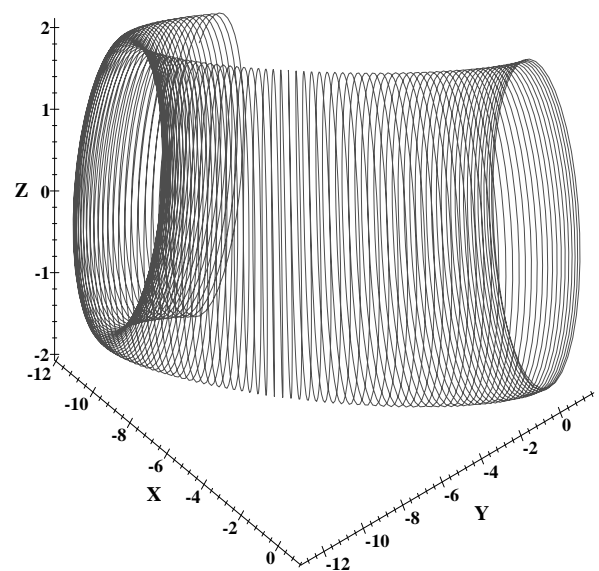

$\mathrm{b}$

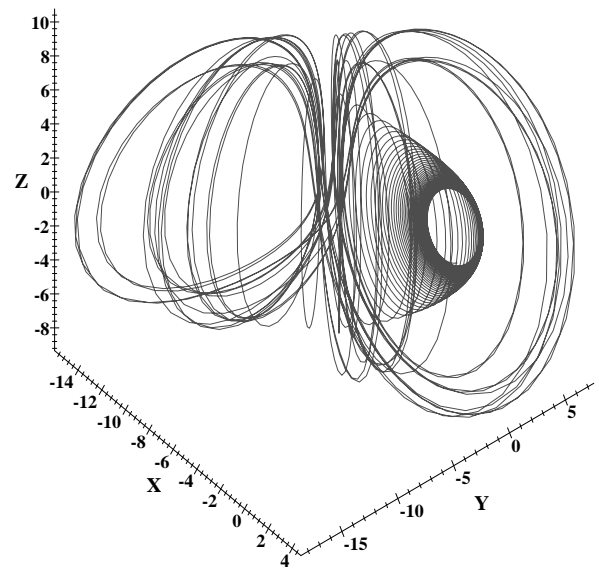

C

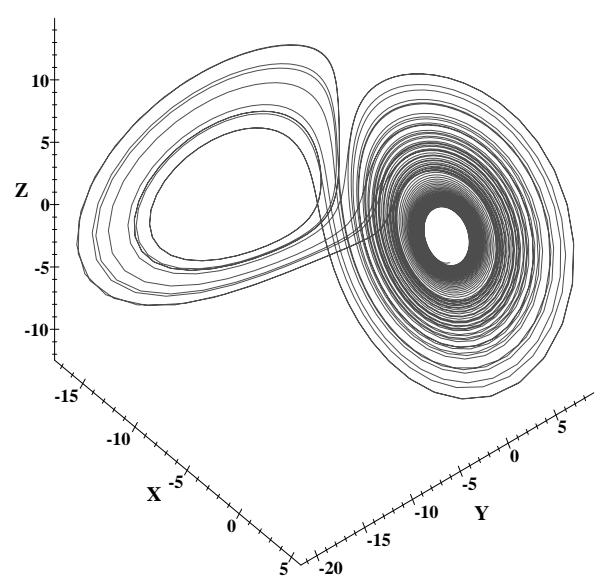

Fig. 2. Trajectories in $3 \mathrm{D} \Re\left(z_{1}\right)-\Re\left(z_{2}\right)-z_{3}$ subspace (X-axis is for $\Re\left(z_{1}\right), \mathrm{Y}$-axis is for $\Re\left(z_{2}\right)$ and Z-axis is for $z_{3}$ ) at $\sigma=5, b=1$ and $r=r_{c}+0.2$ for three different values of the detuning: a) $\Delta=0.5, r_{c} \approx 23.41$; b) $\Delta=0.1, r_{c} \approx 15.28$; c) $\Delta=0.5, r_{c} \approx 15.07$. The plots indicate transition of the attractor from the invariant torus (fig. 1a) to the Lorenz strange attractor (fig. 1c) with a decrease of $\Delta$.

In Sec. 1 the complex Lorenz model was derived from the one-mode laser equations (1), so we need to discuss whether it is possible to observe the above effects exper- 
imentally. Typically, it is difficult to meet the condition $r>r_{c}$ in the one-mode regime and it was just a few experiments with gas lasers, where the threshold of equilibrium state instability was exceeded $[17,18]$. Based on the above theory, in experimental setup of [17] with homogeneously broadened one-mode $\mathrm{CO}_{2}$ laser one could expect three different types of the system behaviour as the pumping increases:

- At sufficiently small detuning $\Delta<\Delta_{0},\left(\Delta_{0} \approx 0.07\right.$ at $\sigma=5$ and $b=1)$ the chaotic attractor forms abruptly during the passage of $r$ through $r_{c}$;

- The system undergoes a cascade of doublings before its transition to chaos at $\Delta_{0}<\Delta<\Delta_{c}$;

- If $\Delta>\Delta_{c}$, the system does not reveal chaotic behaviour even if $r \approx(10-20) r_{c}$.

In conclusion, it is interesting to point out that, following the line of [19], where three parameter kinetics of a phase transition was investigated by using the real Lorenz model, the complex Lorenz model can be used in studying the kinetics of a non-equilibrium second-order phase transition in the case of complex order parameter. From this standpoint, the model (2) can be considered within the synergetic concept of a phase transition [20]. It means that a phase transition is realized as a result of mutual coordination between the complex order parameter $\left(z_{1}\right)$, the conjugate field $\left(z_{2}\right)$ and the control parameter $\left(z_{3}\right)$. The results, given at the beginning of this section, can be regarded as an extension of the analogy between non-equilibrium phase transitions and phase transitions in thermodynamic systems.
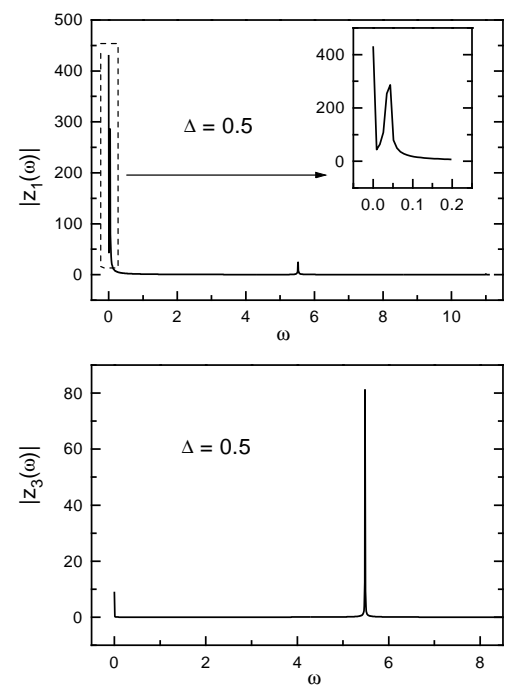

Fig. 3. Fourier spectra $\left|z_{1}(\omega)\right|$ and $\left|z_{3}(\omega)\right|$ at $\sigma=5, b=1$ and $r=r_{c}+0.2$ for $\Delta=0.5$. There are three peaks in the spectrum $\left|z_{1}(\omega)\right|: \omega=0, \omega \approx 0.04$ (see the inset in the upper right corner), $\omega \approx \alpha=5.5$. The second peak, associated with the Goldstone-type mode, is absent in the spectrum $\left|z_{3}(\omega)\right|$.
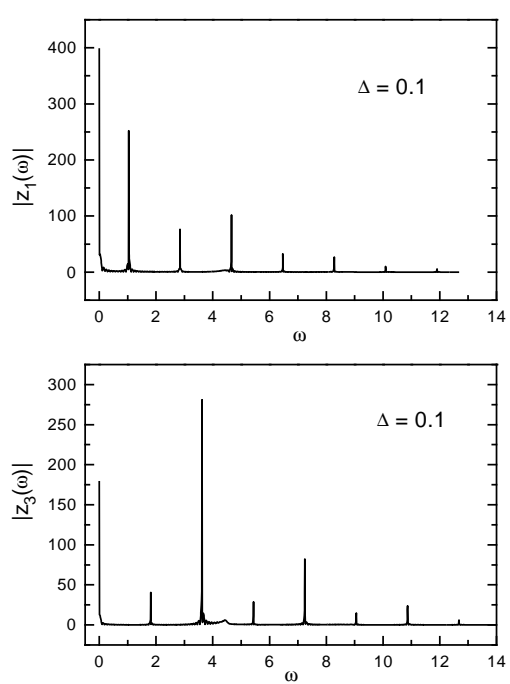

Fig. 4. Fourier spectra $\left|z_{1}(\omega)\right|$ and $\left|z_{3}(\omega)\right|$ at $\sigma=5, b=1$ and $r=r_{c}+0.2$ for $\Delta=0.1$. Both spectra are typical of doublings.

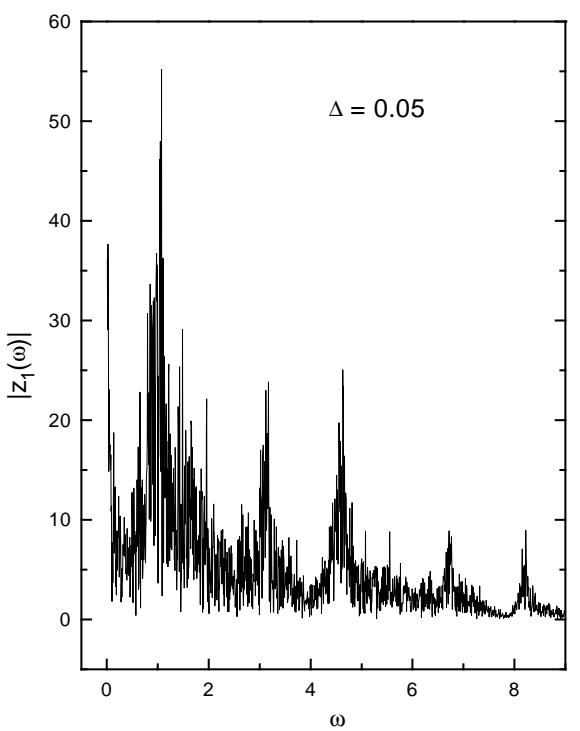

Fig. 5. The irregular (noisy) Fourier spectrum $\left|z_{1}(\omega)\right|$ at $\sigma=5, b=1$ and $r=r_{c}+0.2$ for $\Delta=0.05$.

\section{ACKNOWLEGMENTS}

The authors are grateful to A. I. Olemskoi for his stimulating remarks and valuable discussion. 
[1] E. N. Lorenz, J. Atmos. Sci. 20, 130 (1963).

[2] V. S. Afraimovich, V. P. Bykov, L. P. Shil'nikov, Dokl. Akad. Nauk SSSR 234, 336 (1977).

[3] R. F. Williams, Lect. Notes in Math. 615, 94 (1977).

[4] J. E. Marsden, M. McCracken (eds.), The Hopf Bifurcation and Its Applications (Springer-Verlag, New-York, 1976).

[5] C. Sparrow, The Lorenz Equations: Bifurcations, Chaos and Strange Attractors (Springer-Verlag, Berlin, 1982).

[6] Yu. I. Neimark, P. S. Landa, Stochastic and Chaotic Oscillations (Nauka, Moscow, 1987) (in Russian).

[7] J. B. McLaughlin, P. C. Martin, Phys. Rev. A 12, 186 (1975).

[8] K. A. Robbins, SIAM J. Appl. Math. 36, 457 (1979).

[9] P. Manneville, Y. Pomeau, Phys. Lett. 75 A, 1 (1979).

[10] A. C. Fowler, M. J. McGuinness, Phys. Lett., 92 A, 103 (1982).

[11] A. C. Fowler, J. D. Gibbon, M. J. McGuinness, Physica D 4, 139 (1982).
[12] H. Haken, Laser Light Dynamics (North-Holland Phys. Publishing, 1985)

[13] A. A. Bakasov, Teor. i Matem. Fiz. 89, 278 (1991) (in Russian).

[14] G. Iooss, D. D. Joseph, Elementary Stability and Bifurcation Theory (Springer-Verlag, Berlin, 1980).

[15] D. D. Joseph, Arch. Rational. Mech. Annal. 67, 99 (1977).

[16] G. Iooss, D. D. Joseph, Arch. Rational. Mech. Annal. 66, 135 (1977).

[17] W. Klische, C. O. Weiss, Phys. Rev. A 31, 4049 (1985).

[18] F. T. Arrechi, R. Meucci, G. Puccioni, J. Treduce, Phys. Rev. Lett. 49, 1217 (1982).

[19] A. I. Olemskoi, A. V. Khomenko, Zh. Eksp. Teor. Fiz. 83, 1180 (1996).

[20] H. Haken, Advanced Synergetics, Instability Hierarchies of Self-Organizing Systems and Devices (Springer, Berlin, 1987).

\title{
ПОРУШЕННЯ СИМЕТРІЇ Й БІФУРКАЦІЇ В КОМПЛЕКСНІЙ МОДЕЛІ ЛОРЕНЦА
}

\author{
О. Д. Кисельов \\ Чернігівсъкий технологічний інститут, кафедра вищої та прикладної математики, \\ вул. Шевченка, 95, Чернігів, UA-250027, Україна \\ E-mail:kisel@elit.chernigov.ua; adk@kid.ti.chernigov.ua
}

\begin{abstract}
Використано концепцію порушеної симетрії для вивчення стійкости стаціонарних та двічі періодичних у часі біфуркаційних розв'язків комплексної моделі Лоренца на підставі модифікованої теорії Хопфа залежно від відстроювання частоти. На відміну від добре відомих рівнянь Лоренца, ця система є інваріянтною щодо перетворень групи Лі (обертання в комплексних площинах). Знайдено, що біфуркація іваріянтної множини стаціонарних точок приводить до утворення інваріянтного тору, якщо відстроювання перевищує його критичне значення. Результати числового аналізу показали, що при зменшенні відстроювання після каскаду біфуркацій подвоєння періоду в околі точного резонансу утворюється дивний атрактор Лоренца.
\end{abstract}

